\title{
The Diversification Delta: A Different Perspective
}

\author{
Yuri Salazar Flores, Robert J. Bianchi, Michael E. Drew, Stefan Trück
}

Yuri Salazar Flores is a Lecturer at the Faculty of Sciences, National Autonomous University of Mexico, Mexico City, Mexico.

yurisf@ciencias.unam.mx

Robert J. Bianchi is an Associate Professor of Finance at Griffith Business School and Director of the Griffith Centre for Personal Finance and Superannuation at Griffith University in Brisbane, QLD, Australia.

r.bianchi@griffith.edu.au

Michael E. Drew is a Professor of Finance at Griffith Business School at Griffith University in Brisbane, QLD, Australia.

michael.drew@griffith.edu.au

Stefan Trück is a Professor of Finance at the Faculty of Business and Economics and Co-Director of the Centre for Financial Risk at Macquarie University in Sydney, NSW, Australia.

stefan.trueck@mq.edu.au

\begin{abstract}
In a 2012 article published in this Journal, Vermorken, Medda, and Schröder introduce a new measure of diversification, the Diversification Delta (DD), based on the entropy of the portfolio return distribution. Entropy as a measure of uncertainty has been used successfully in several frameworks and takes into account the entire statistical distribution rather than just the first two moments. In this article, the authors highlight some drawbacks of the DD measure and go on to propose an alternative measure based on exponential entropy which overcomes the identified shortcomings. The authors present the properties of this new measure and propose it as an alternative for portfolio optimization that incorporates higher moments of asset returns such as skewness and excess kurtosis.
\end{abstract}


Vermorken et al. (2012) introduce a new measure of diversification, which is based on higher moments of the return distribution of a portfolio. The proposed measure, called Diversification Delta (DD), is based on the concept of Shannon entropy, or information entropy, that can measure the uncertainty related to the entire statistical distribution and not just the first two moments of a distribution.

Investors typically diversify their portfolios with the aim of reducing their exposure to idiosyncratic risks of individual assets, while the correlation matrix of asset returns is regarded as the common metric for measuring portfolio diversification. However, the correlation matrix quantifies the pairwise relation between two or more stochastic processes. As pointed out by Statman and Scheid (2008), the correlation matrix does not account for the impact of individual assets on the variance of the portfolio. Furthermore, modern portfolio theory quantifies the level of diversification by using only the first two moments of the return distribution.

As stated in Vermorken et al. (2012), different diversification measures have been proposed in the finance literature. Various researchers consider the use of the correlation matrix, as well as alternative measures such as clustering based methods, the portfolio diversification index, and the return gaps. For a detailed analysis of these methods, see, for example, Dopfel (2003), Brown and Goetzmann (2003), Rudin and Morgan (2006), and Statman and Scheid (2008). These models come as a response to the classic portfolio optimization model introduced by Markowitz (1952). Other models include indexes based on mean-variance analysis (MVA) such as the reward-to-variability ratio by Sharpe (1966), hereafter referred to as SR, and indexes based on risk measures, such as the diversification index defined by Tasche (2006), which is based on the Value-at-Risk (VaR). Although the use of these indexes has been successful to a large extent, the DD has the advantage of not being restricted to the first two moments of the return distribution. It also captures diversification by comparing return distributions of the assets before and after the portfolio is constructed. At the same time, it is relatively easy to calculate and interpret.

As argued by Vermorken et al. (2012), entropy captures the reduction in uncertainty as a portfolio of various assets becomes more diversified. More diversification reduces uncertainty and lowers entropy. The proposed DD measure has the advantage of being a straightforward application and interpretation for portfolios consisting of different asset classes. Based on an empirical example and using returns from different infrastructure indexes, the authors argue that the application of DD '[..] gives the portfolio manager a much 
clearer picture of the reality in the market than the correlation coefficient'. Therefore, DD presents an interesting contribution to the study of portfolio theory and diversification.

However, the construction of the DD measure based on the exponential of the weighted mean of the entropies of the individual assets leads to some issues that will be examined in this study. For example, it is easy to illustrate that the proposed measure does not provide a range of $0 \leq \mathrm{DD} \leq 1$ as suggested by Vermorken et al. (2012). This is true, in particular, when assets with a different level of risk or variance are combined, which makes an accurate interpretation of the measure quite difficult. Further, in some instances, the measure provides results that contradict what one would intuitively expect from a diversification measure. We propose a revised measure that uses the weighted mean of the exponential entropies of the individual assets in a portfolio as a suitable alternative.

The remainder of the paper is structured as follows. We first analyze the DD presented in Vermorken et al. (2012) and illustrate some shortcomings of this measure. We then present a revised measure that is also based on the concept of Shannon entropy of a random variable but overcomes the problems of the original DD. We then illustrate how higher moments such as skewness and kurtosis impact portfolios that are constructed when using the new DD measure. The conclusion summarizes our main findings and discusses the application of the new DD measure in portfolio management.

\section{The Diversification Delta}

The DD is based on the entropy as a measure of uncertainty. Originally, entropy was defined in a statistical mechanics framework and introduced in information theory by Shannon (1948). Since then, entropy has been successfully used in measuring uncertainty in several areas such as applied mathematics, electrical engineering, computer science, physics, neuroscience, among others.

In econometrics, discrete entropy is often maximized to fit probability functions, for example, in the work of Maasoumi (1993) and Ullah (1996). In the context of financial economics, entropy and conditional entropy have been used to define convex risk measures (Laeven and Stadje, 2010; Föllmer and Knispel, 2011), while entropy measures have been used to estimate distributions associated with financial data (Kitamura and Stutzer, 1997; Robertson et al., 2005).

Although entropy is not a widespread measure in the portfolio optimization literature or among practitioners, studies and applications exist. In the seminal work of Philippatos and 
Wilson (1972), entropy is used for the first time in a portfolio optimization framework. Following this, Hua and Xingsi (2003) and Bera and Park (2008) report that entropy can be an effective alternative to the MVA. More recently, entropy has received new attention because it can capture heavy tails that are often present in financial return data (Urbanowicz et al., 2012; Dey and Juneja, 2012). Regarding the use of the concept of entropy to build diversification measures, Bera and Park (2008) and Meucci (2009) develop diversification measures based on maximizing entropy.

The framework by which entropy is used in the definition of the DD differs from the way it is used in other portfolio analysis methods. In this case, entropy plays the central role and is evaluated in both the assets and the portfolio with no further analysis needed. Also, as suggested by Campbell (1966), exponential entropy is used to avoid singularities of entropy, while still letting the uncertainty 'speak for itself.' Exponential entropy is also mentioned as a risk measure in Rachev et al. (2012).

For a given portfolio $P$ consisting of $N$ assets $\left(X_{1}, \ldots, X_{N}\right)$ and weights $\left(w_{1}, \ldots, w_{N}\right)$, with $\sum_{i=1}^{N} w_{i}=1$, Vermorken et al. (2012) define the DD as

$$
D D(P)=\frac{\exp \left(\sum_{i=1}^{N} w_{i} H\left(X_{i}\right)\right)-\exp \left(H\left(\sum_{i=1}^{N} w_{i} X_{i}\right)\right)}{\exp \left(\sum_{i=1}^{N} w_{i} H\left(X_{i}\right)\right)}
$$

where $f$ is the density of $X$ and $H(X)=-\int_{x}[f(x)] \log (f(x)) d x$ is the differential entropy that is used as a measure of uncertainty. The estimator of entropy they consider is the one developed in Stowell and Plumbley (2009). The higher the level of entropy, the higher the uncertainty and vice-versa. The DD is designed to measure the diversification effect of a portfolio by considering entropy of the individual assets and by comparing it with the entropy of the portfolio.

The DD is defined as a ratio that compares the weighted individual assets and the portfolio. The value of using such a ratio is that it quantifies the effect of portfolio diversification. Furthermore, using entropy to measure uncertainty in this context is itself an important contribution. However, the way entropy is used by Vermorken et al. (2012) to measure uncertainty presents some issues. First, the measure of uncertainty in Equation (1) must satisfy a number of properties for the ratio to adequately measure portfolio 
diversification. Artzner et al. (1999), Rockafellar et al. (2006), and McNeil et al. (2005), among others, have analyzed desirable properties for measures of risk and uncertainty in a portfolio optimization framework. Although entropy has proven to be effective in measuring uncertainty, it is understood that it is not homogeneous, is not left-bounded, and is not subadditive, as noted by Cover and Thomas (1991). In the definition of the DD, the exponential function is used to account for these issues; however, it is not achieved in an adequate framework, as we now discuss in further detail.

Recalling that the DD compares the individual assets with the portfolio using a measure of uncertainty, the first characteristic we notice is that, for the DD to be positive, the measure of uncertainty must satisfy some kind of subadditivity. This will ensure that the numerator in (1) is positive. As noted by Artzner et al. (1999) and McNeil et al. (2005), subadditivity is a crucial feature for measures of uncertainty or risk because it reflects the fact that risk can be reduced by diversification. The authors also comment on the shortcomings of the use of measures that do not account for this, especially VaR (McNeil et al., 2005). Subadditivity is not satisfied in the definition of the DD, which can, in fact, be negative and is also not left-bounded.

Vermorken et al. (2012) state that the DD should exhibit a range of outcomes between 0 and 1 . The authors suggest that this is actually one of the great benefits of the measure allowing for straightforward interpretation. While a value of 0 represents no diversification, a '[...] value of one indicates that only market risk remains in the portfolio and all idiosyncratic risk has been diversified' (page 68). Therefore, the measure is not meant to be negative, since this causes some difficulties with respect to its interpretation. As we will illustrate in an example, negative outcomes for DD are likely to occur even under very plausible scenarios. These negative values are problematic because they contravene the essential idea of the DD.

Homogeneity is another desirable property for measures of uncertainty because it ensures that changes in the size of an asset or the portfolio are detected according to their magnitude ${ }^{1}$. Given that $H(a X)=H(X)+\log (|a|)$, the left-hand side in the numerator of (1) is not homogeneous with respect to the asset, see, e.g., Cover and Thomas (1991). This means that changes in the size of the assets are not detected in the same way as changes in the portfolio, leading to inconsistencies in the measurement of diversification. In the following Examples 1

\footnotetext{
${ }^{1}$ A function $F$ is homogenous if for a constant $a, F(a x)=a F(x)$.
} 
to 3, we highlight the shortcomings of the DD we have described. For simplicity, we consider the bivariate case.

In Example 1, we present a portfolio formed by two assets following a normal distribution. We consider the particular case when one of the assets is riskier than the other, indicated by a significantly higher variance. Such a scenario is realistic for real world portfolios, for example when combining defensive assets (bonds) with growth assets (equities). Unfortunately, Vermorken et al. (2012) in their analysis do not consider such a case, but rather focus on the behavior of the DD when assets with identical or similar variance are combined to create a portfolio.

Example 1 Assume that the expected returns of Asset 1 are normally distributed with $\mu_{X_{1}}=0.05$ and $\sigma_{X_{1}}=0.1$, while expected returns of Asset 2 follow a normal distribution with $\mu_{X_{2}}=0.01$ and $\sigma_{X_{2}}=0.02$.

Thus, the first asset yields a higher expected return with higher risk, measured by the standard deviation. The second asset exhibits a lower expected return, but also a significantly lower standard deviation. The DD for a portfolio with normally distributed assets can be expressed as (see Appendix A, equation (6) of the online supplementary materials):

$$
\begin{aligned}
D D(P) & =\frac{\sigma_{X_{1}}^{w_{1}} \sigma_{X_{2}}^{w_{2}}-\sigma_{P}}{\sigma_{X_{1}}^{w_{1}} \sigma_{X_{2}}^{w_{2}}} \\
& =1-\frac{\sigma_{P}}{\sigma_{X_{1}}^{w_{1}} \sigma_{X_{2}}^{w_{2}}} .
\end{aligned}
$$

Let us first consider the case when asset returns are independent. For a portfolio consisting of Asset 1 and Asset 2, the DD is then equal to:

$$
D D(P)=1-\frac{1}{2} \sqrt{\frac{26}{25} \cdot \frac{5}{1}} \approx-0.14
$$

which is less than zero. Furthermore, it is clear that $\operatorname{DD}(P)$ becomes inreasingly negative the smaller $\sigma_{X_{2}}$ is. In fact, if $\sigma_{X_{2}} \rightarrow 0$, then $\sigma_{P} \rightarrow w_{1} \sigma_{X 1}=0.1 w_{1}$ and, from equation (6), $D D(P) \rightarrow 1-\frac{0.1 w_{1}}{0.1 \cdot 0}=-\infty$. 

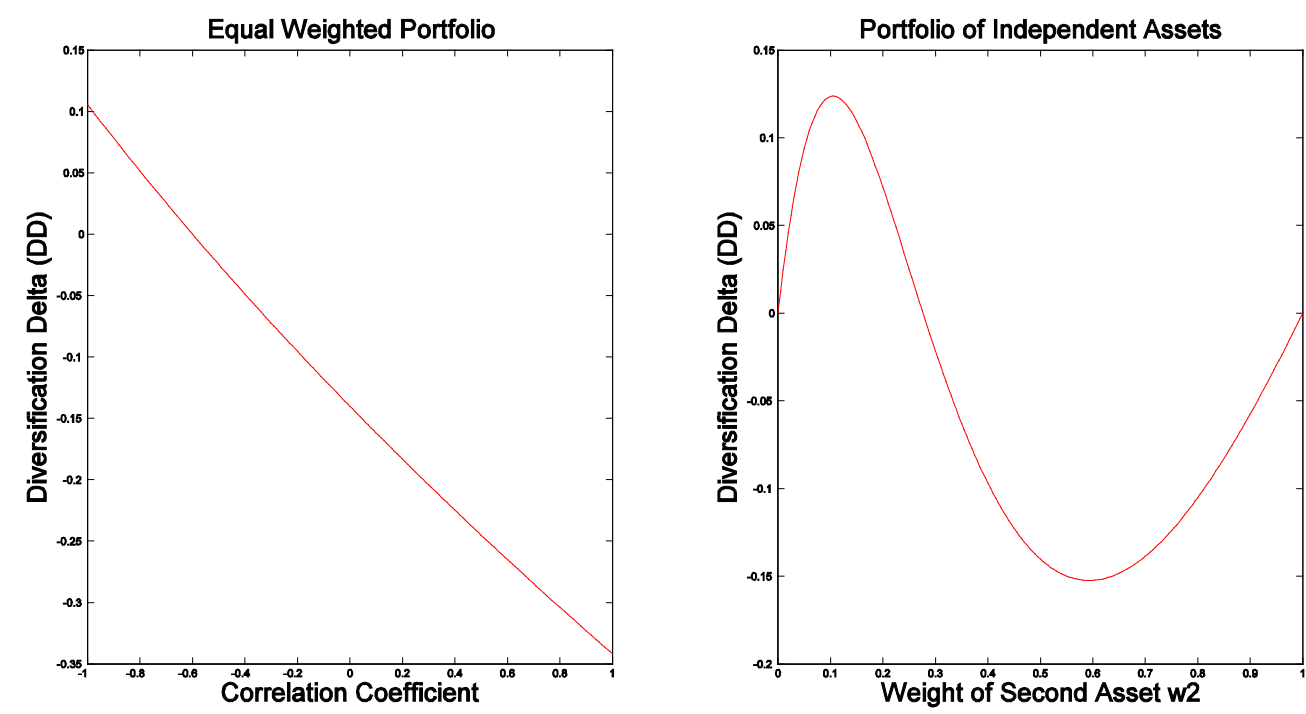

Exhibit 1: Diversification Delta as a function of the correlation coefficient and the portfolio weight $w_{2}$ for an exemplary two-asset portfolio. Expected returns of Asset 1 are normally distributed with $\mu_{X_{1}}=0.05$ and $\sigma_{X_{1}}=0.1$, while expected returns of Asset 2 follow a normal distribution with $\mu_{X_{2}}=0.01$ and $\sigma_{X_{2}}=0.02$. On the left-hand side, we assume $w_{1}=w_{2}=0.5$ and an ex ante determined correlation coefficient varying between -1 and 1 . On the right-hand side, we assume the assets are independent and an ex ante determined weight $w_{2}$ of the second asset.

This issue presents complications when interpreting the DD. Exhibit 1 illustrates the results for the constructed portfolios using Asset 1 and Asset 2. On the left-hand side, similar to Vermorken et al. (2012), we consider an equal weighted portfolio and determine the coefficient of correlation ex ante from -1 to 1 . On the right-hand side we consider a portfolio consisting of independent assets by changing the weight $w_{1}$ of Asset 1 from 0 to 1 . The exhibit illustrates that the DD becomes negative once the coefficient of correlation is greater than -0.8 . It is noteworthy that we still observe that the DD declines once the correlation coefficient increases, i.e., when the diversification is reduced. Therefore, in a relative way, the proposed measure still conveys information on the benefits of diversification when the two assets are combined into a portfolio. However, the interpretation of DD becomes significantly more difficult in this case, since it can take on negative values. The right-hand side shows that the DD exhibits erratic behavior when the weights change, which further complicates its interpretation.

Moreover, as discussed earlier, the lack of homogeneity reduces the efficacy of the DD. It is a well-known fact that portfolios consisting of assets that are a linear combination of one or more assets offer no diversification. However, the DD fails to detect this simple property and 
yields negative values for such portfolios. For simplicity, we will once more consider the bivariate case in our second example and construct a portfolio of assets which are a linear combination of each other.

Example 2 Let $P$ be a portfolio consisting of two assets, $X$ and $a X$, where a denotes a positive constant. Using the property of differential entropy, $H(a X)=H(X)+\log (|a|)$, and $w_{1}+w_{2}=1$, the diversification delta is:

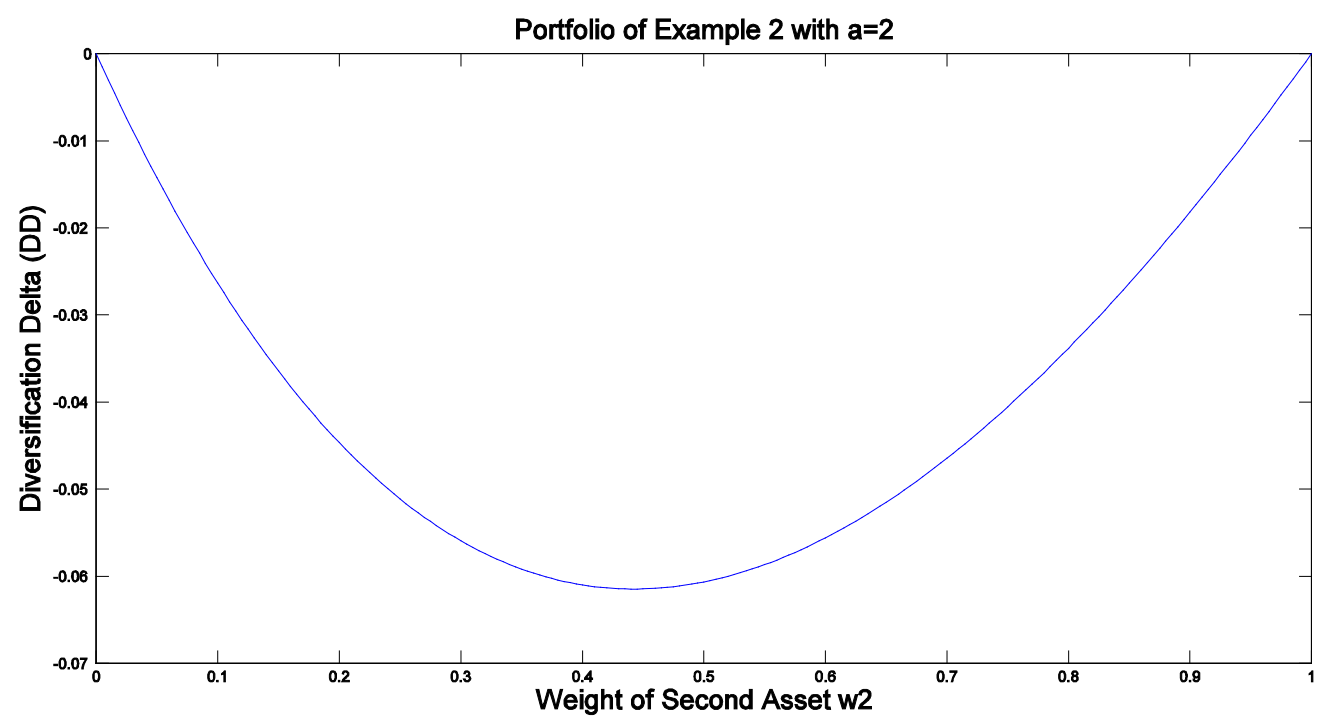

Exhibit 2: Diversification Delta as a function of portfolio weight $w_{2}$ for an exemplary two-asset portfolio with Asset 2 equal to $2^{*}$ Asset 1 . The constructed portfolio will not provide any diversification.

$$
D D(P)=\frac{a^{w_{2}}-\left(1+(a-1) w_{2}\right)}{a^{w_{2}}} .
$$

This value does not depend on entropy $H(X)$ and is only zero when one of the weights is zero and negative in any other case.

Consider Example 2, which describes the case where $a=2$, that is, one of the assets simply equals two times the other asset. In this case the DD can be calculated as $D D(P)=\frac{2^{w_{2}}-\left(1+w_{2}\right)}{2^{w_{2}}}$. In Exhibit 2, we illustrate this case when the weight for Asset 2, $w_{2}$, is allowed to vary from $w_{2}=0$ up to $w_{2}=1$. It becomes obvious that for the illustrated 
example, the DD is negative for all cases except for either $w_{2}=0$ or $w_{2}=1$, where the DD takes on a value of zero. The measure reaches its minimum value for $w_{2}=0.4427$. However, while the constructed portfolio does not provide any diversification benefits, it is also neither less nor more diversified than the original assets 1 or 2 . It is the lack of homogeneity in the left-hand side of the numerator of (1) which yields different results for different weights. For the specified example, an appropriate measure for diversification of a portfolio should be 0 for all constructed portfolios and should not depend on the choice of the portfolio weights.

Let us finally consider Example 3, where we illustrate the lack of homogeneity of the original DD, which leads to inconsistent results.

Example 3 Investor 1 is constructing a portfolio consisting of assets $X_{1}$ and $X_{2}$. This investor determines that a portfolio with equal weights is optimal, i.e,. $P_{1}=\frac{1}{2} X_{1}+\frac{1}{2} X_{2}$. In a different market, investor 2 constructs a portfolio from assets $Y_{1}=\frac{3}{2} X_{1}$ and $Y_{2}=\frac{3}{4} X_{2} \cdot{ }^{2}$ This investor determines optimal weights of $w_{1}=\frac{1}{3}$ and $w_{2}=\frac{2}{3}$, yielding the same portfolio $P_{2}=\frac{1}{2} X_{1}+\frac{1}{2} X_{2}$. Given that both portfolios are the same and have the same underlying assets, one would expect that the two portfolios have the same DD. However, this is not the case (see Appendix B of the online supplementary materials) such that

$$
D D\left(P_{1}\right) \neq D D\left(P_{2}\right)
$$

This follows from the lack of homogeneity of the left-hand side of the numerator in equation (1).

Given the drawbacks we identified in the DD, we now define an alternative measure.

\section{A revised Diversification Delta $\left(D D^{*}\right)$ measure}

As we stated before, using a ratio that compares the uncertainty of individual assets with the uncertainty of the portfolio is an interesting approach to portfolio analysis. Also, given the ability of entropy to measure uncertainty while taking into account higher moments, we agree with Vermorken et al. (2012) that a measure based on entropy will provide a useful tool to quantify portfolio diversification. We now focus on the issue of defining a measure that

${ }^{2}$ Note that the coefficients in these equations represent volume, not weights. 
overcomes the drawbacks of the original DD, while still relying on entropy to measure uncertainty. As we have seen, a measure of uncertainty should satisfy certain properties in order to be well defined. In particular, it is desirable that the measure is homogeneous and subadditive, see Artzner et al. (1999); McNeil et al. (2005). The measure should also be bounded between 0 and 1, while reflecting the level of portfolio diversification.

Differential entropy in Vermorken et al. (2012) is not subadditive or homogeneous. Moreover, unlike discrete entropy, it can be negative. Differential entropy is maximized by the normal distribution. That is, when $X$ is a random variable with finite variance $\sigma_{X}^{2}$, we have:

$$
H(X) \leq \log \left(\sqrt{2 \pi e \sigma_{X}^{2}}\right)
$$

see, for example, Cover and Thomas (1991).

An interesting case for analysis is the entropy of a constant, that is when $X=C$ and $\sigma_{X}=0$. Although the density is not defined in this case, it is obvious that when a random variable tends to approach a constant, its differential entropy tends to approach $-\infty$. Although this is consistent with the idea that the smaller the entropy, the less uncertainty there is, it makes entropy more difficult to deal with.

These issues can be addressed by considering exponential entropy as a measure of uncertainty. Exponential entropy satisfies the following properties for variables $X$ and $Y$ and constants $C \in \mathbb{R}$ and $\lambda>0,{ }^{3}$

(1) $\exp (H(X+C))=\exp (H(X))$,

(2) $\exp (H(0))=0$ and $\exp (H(\lambda X))=\lambda \exp (H(X))$,

(3) $\exp (H(X+Y)) \leq \exp (H(X))+\exp (H(Y))$,

These properties imply that for random variables $X_{1}, \ldots, X_{N}$ and weights $\left(w_{1}, \ldots, w_{N}\right)$, with $\sum_{i=1}^{N} w_{i}=1$ :

$$
\exp \left(H\left(\sum_{i=1}^{N} w_{i}\left(X_{i}\right)\right)\right) \leq \sum_{i=1}^{N} w_{i} \exp \left(H\left(X_{i}\right)\right)
$$

${ }^{3}$ For a proof, see Cover and Thomas (1991). 
This is straightforward for $N=2$ and it follows inductively for higher values. Considering this and properties (1) to (3), we propose the following revised measure of the DD, that is $D D^{*}$ of a portfolio $P$ :

$$
D D^{*}(P)=\frac{\sum_{i=1}^{N} w_{i} \exp \left(H\left(X_{i}\right)\right)-\exp \left(H\left(\sum_{i=1}^{N} w_{i} X_{i}\right)\right)}{\sum_{i=1}^{N} w_{i} \exp \left(H\left(X_{i}\right)\right)} .
$$

We note that the difference with the original DD proposed by Vermorken et al. (2012) is that we use the weighted mean of the exponential entropies of the individual assets, instead of the exponential of the weighted mean of the entropies of the individual assets, on the left-hand side of the numerator and in the denominator. Note that like in Vermorken et al. (2012), the estimator of the entropy $H(x)$ we consider is also the one found in Stowell and Plumbley (2009). We now analyze Examples 1 to 3 using the new $D D^{*}$ measure. 

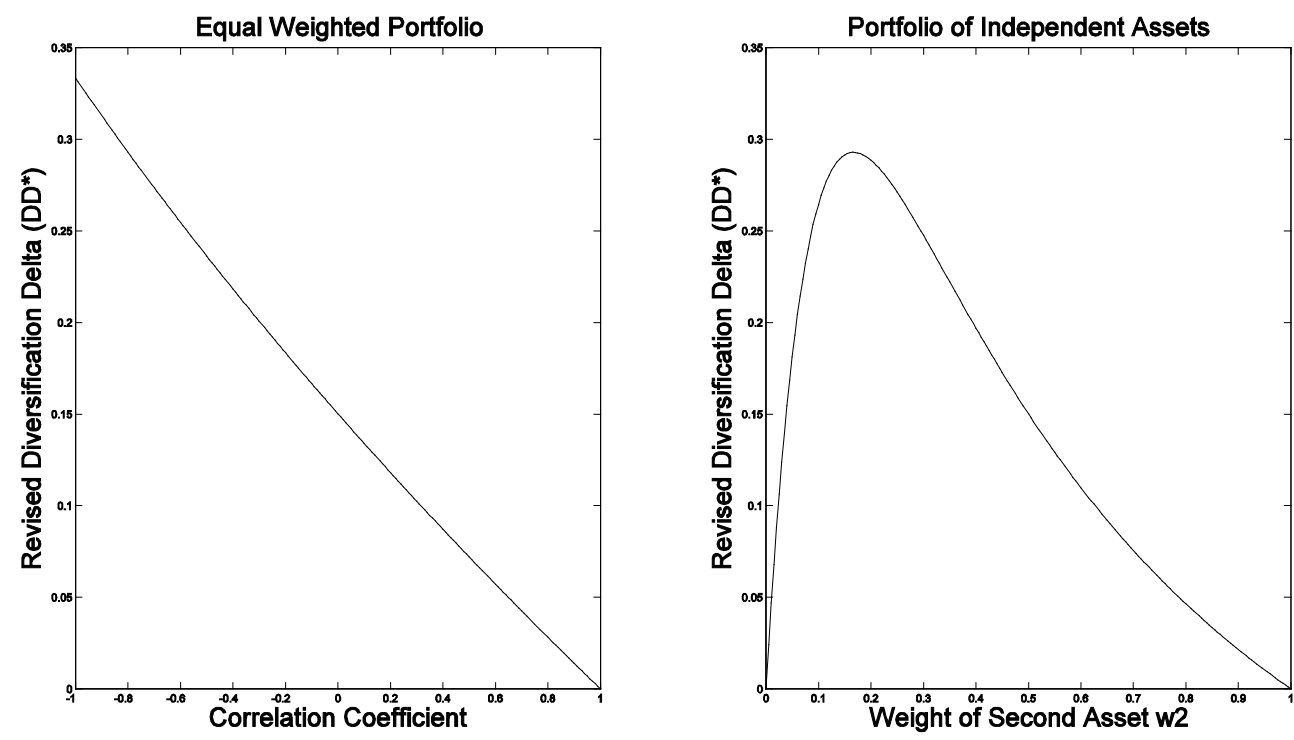

Exhibit 3: New Diversification Delta $D D^{*}$ as a function of the coefficient of correlation and portfolio weight $w_{2}$ for an exemplary two-asset portfolio. Expected returns of Asset 1 are normally distributed with $\mu_{X_{1}}=0.05$ and $\sigma_{X_{1}}=0.1$, while expected returns of Asset 2 follow a normal distribution with $\mu_{X_{2}}=0.01$ and $\sigma_{X_{2}}=0.02$ . On the left-hand side, we assume $w_{1}=w_{2}=0.5$ and an ex ante determined correlation coefficient varying between -1 and 1 . On the right-hand side, we assume the assets are independent and an ex ante determined weight of the second asset.

\section{Examples 1 to 3 revisited.}

Considering Example 1, in the bivariate Gaussian case, the new $D D^{*}$ is equal to ${ }^{4}$

$$
\begin{aligned}
D D^{*}(P) & =\frac{\left(w_{1} \sigma_{X_{1}}+w_{2} \sigma_{X_{2}}\right)-\sigma_{P}}{\left(w_{1} \sigma_{X_{1}}+w_{2} \sigma_{X_{2}}\right)} \\
& =1-\frac{\sigma_{P}}{\left(w_{1} \sigma_{X_{1}}+w_{2} \sigma_{X_{2}}\right)},
\end{aligned}
$$

with $\sigma_{P}^{2}=w_{1}^{2} \sigma_{X_{1}}^{2}+2 \rho w_{1} w_{2} \sigma_{X_{1}} \sigma_{X_{2}}+w_{2}^{2} \sigma_{X_{2}}^{2}$. Note that only when the variance for Asset 1 and Asset 2 are the same, the new measure and the original DD in Equation (2) are identical and equal to $1-\frac{\sigma_{P}}{\sigma_{X_{1}}}$. However, in Appendix $\mathrm{A}$ of the online supplementary materials, we illustrate that the two measures will yield significantly different results when $\sigma_{X_{1}} \neq \sigma_{X_{2}}$. Thus, for Example 1, with $\sigma_{X_{1}}=0.1$ and $\sigma_{X_{2}}=0.02$ we obtain quite different results for the original and the revised measure. In Exhibit 3 we replicate Exhibit 1 using the new

\footnotetext{
${ }^{4}$ See Appendix A, equation (7) of the online supplementary materials.
} 
diversification measure $D D^{*}$. We find that all values for $D D^{*}$ are between 0 and 1 and exhibit a less erratic behavior in comparison to the original DD.

Now, considering Example 2, let $P$ be a portfolio consisting of assets $\left(X_{1}, \ldots, X_{N}\right)$ which are all positive linear combinations of an asset $X$. Hence, $X_{i}=a_{i} X+b_{i}$, for constants $a_{i}>0$ and $b_{i}$ with $i \in\{1, \ldots, N\}$. Using the properties of differential entropy it is easy to show (see Appendix $\mathrm{C}$ of the online supplementary materials) that

$$
\sum_{i=1}^{N} w_{i} \exp \left(H\left(X_{i}\right)\right)=\exp \left(H\left(\sum_{i=1}^{N} w_{i} X_{i}\right)\right),
$$

such that the revised measure of diversification takes on a value of zero in this case. Therefore, the revised measure will be equal to zero for this case, where no diversification is achieved, since only linear combinations of an individual asset $X$ are being combined.

Finally, for Example 3, let us consider the same notations as previously, in particular $c_{1}=\exp \left(H\left(X_{1}\right)\right), \quad c_{2}=\exp \left(H\left(X_{2}\right)\right)$ and $c_{3}=\exp \left(H\left(\frac{1}{2} X_{1}+\frac{1}{2} X_{2}\right)\right)$. It is easy to show (see Appendix D of the online supplementary materials) that for the new measure, we get

$$
D D^{*}\left(P_{1}\right)=D D^{*}\left(P_{2}\right)=\frac{c_{1}+c_{2}-2 c_{3}}{c_{1}+c_{2}}
$$

Given the homogeneity of exponential entropy, the same holds for similar constructions. From the analysis, the $D D^{*}$ measure is always between 0 and 1 , therefore, it is equal to 1 when the portfolio is constant (no risk) and 0 when the assets are a positive linear combination of a single asset (perfect positive dependence). Also, given the homogeneity of exponential entropy, changes in the size of the portfolio and the assets are detected according to their magnitude.

\section{Diversification Delta and the Sharpe Ratio}

Let us now consider how the original DD and the newly derived diversification measure $D D^{*}$ relate to the SR. Assume that the expected returns of Asset 1 are normally distributed with $\mu_{1}=0.05$ and $\sigma_{1}=0.1$, while expected returns of Asset B follow a normal distribution with $\mu_{2}=0.01$ and $\sigma_{2}=0.02$. So the first asset yields a higher expected return with higher risk, 

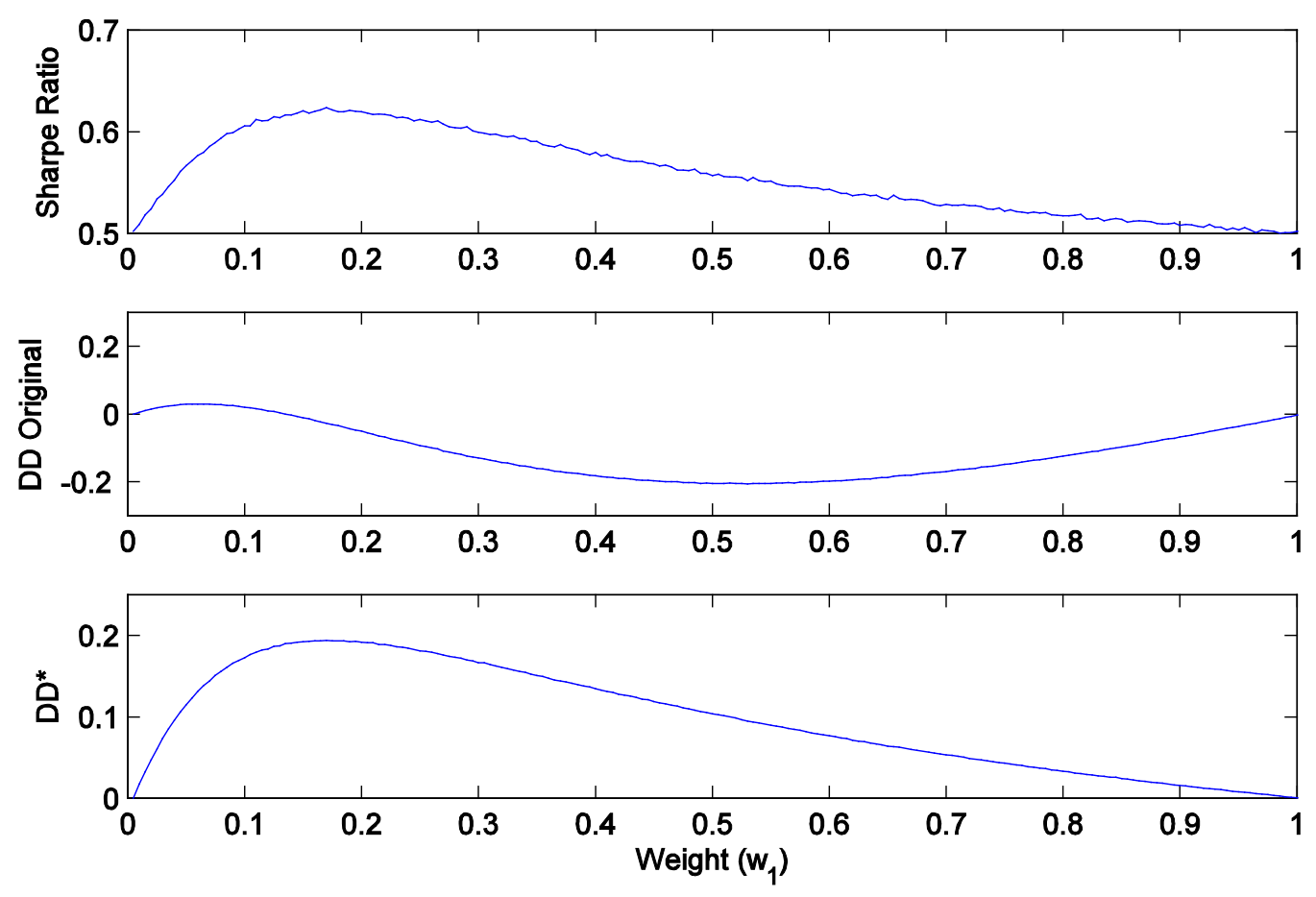

Exhibit 4: Sharpe ratio (upper panel), original Diversification Delta (DD) (middle panel) and revised Diversification Delta $D D^{*}$ (lower panel) as a function of the coefficient of portfolio weight $w_{1}$ for the exemplary two-asset portfolio. Expected returns $r_{1}$ of Asset 1 are normally distributed with $\mu_{X_{1}}=0.05$ and $\sigma_{X_{1}}=0.1$, while returns of Asset 2 are normally distributed with $\mu_{X_{2}}=0.01$ and $\sigma_{X_{2}}=0.02$. Asset 1 and Asset 2 are combined into a portfolio with $w_{1}+w_{2}=1$. The coefficient of correlation between the returns of Asset 1 and Asset 2 is set at 0.3. Both the $D D^{*}$ and the Sharpe ratio are maximized for $w_{1}=0.175$

measured by the standard deviation, while the second asset has a lower expected return, but also a significantly lower standard deviation. We assume that the coefficient of correlation equals $\rho=0.3$ for this example and that the portfolio consists only of these two assets, i.e., $w_{1}+w_{2}=1$. We now calculate values of the $\mathrm{SR}^{5}$, the original $\mathrm{DD}$, and the revised $D D^{*}$ for portfolios in which $w_{1}$ and $w_{2}$ are determined ex ante. In other words, we express the considered diversification and SR measures as a function of $w_{1}$, the weight for the riskier asset.

Exhibit 4 once more illustrates that the original DD does not contain any information that can be easily interpreted by an investor. The measure takes on negative values for $0.15 \leq w_{1} \leq 1$ which makes it almost impossible to create a meaningful interpretation. On the other hand, we observe that for normally distributed returns, the SR and the revised $D D^{*}$ are

${ }^{5}$ Note that for simplification, in our Sharpe ratio calculations, we assume a zero risk-free rate. 
highly correlated. This makes sense, since entropy of the normal distribution is entirely defined by its variance; therefore, a combination of assets with the highest SR will also yield a high $D D^{*}$. Both measures are initially rising to their maximum value for $w_{1}=0.175$ and then they decline. Since both individual assets provide an SR of 0.5, this is the lowest possible outcome for the ratio when either $w_{1}=1$ or $w_{1}=0$. Overall, we find that for the different portfolios we obtain $0.5 \leq S R \leq 0.6219$. The revised $D D^{*}$, on the other hand, takes on a value of 0 when the entire investment is either allocated to Asset 1 or Asset 2 (no diversification) and increases when the two assets are combined. It reaches a maximum value for $w_{1}=0.175$ where we obtain $D D^{*}=0.1938$.

Overall, we conclude that when returns follow a normal distribution, $D D^{*}$ and the SR yield similar results for the portfolio that maximizes these measures. However, unlike the SR, the $D D^{*}$ considers the variance of the different assets (and not only of the portfolio) and is not dependent on the expected return. In that sense, the $D D^{*}$ measures whether the risk is diversified away by the portfolio and depends only on risk. It must be emphasized that real-world asset returns are not normally distributed but will exhibit skewness, excess kurtosis, and other features that make the empirical return distribution deviate from a normal distribution. Therefore, under real-world scenarios, the SR and $D D^{*}$-optimal portfolios may be different. In the following, we will examine the behavior of the $D D^{*}$ measure for skewed and leptokurtic returns in more detail.

\section{Higher Moments}

We will now investigate how changes in higher moments of the return distribution impact the proposed $D D^{*}$ measure. So far, the analysis has been restricted to assets with symmetric returns from a Gaussian distribution. Since the proposed $D D^{*}$ is based on the concept of Shannon entropy, it should take into account the uncertainty related to the entire return distribution and not just the first two moments of the distribution. Therefore, we examine the behavior of $D D^{*}$ for different levels of skewness and kurtosis for an exemplary two-asset portfolio.

We consider two assets similar to the previous examples: expected returns of Asset 1 are distributed with $\mu_{X_{1}}=0.05$ and $\sigma_{X_{1}}=0.1$, while expected returns of Asset 2 follow a distribution with $\mu_{X_{2}}=0.01$ and $\sigma_{X_{2}}=0.02$. We first examine a portfolio with equal 

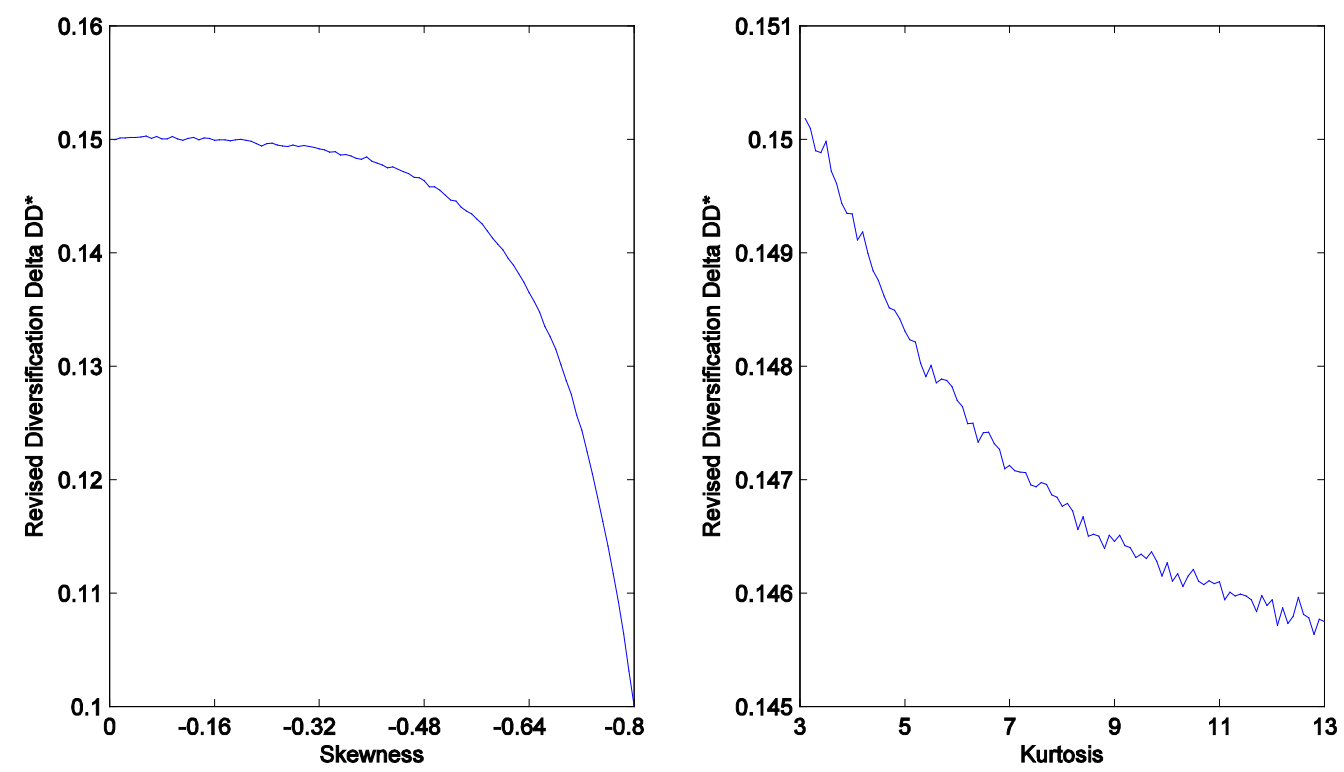

Exhibit 5: New Diversification Delta $D D^{*}$ as a function of skewness and kurtosis for an exemplary two-asset portfolio. Expected returns of Asset 1 are distributed with $\mu_{X_{1}}=0.05$ and $\sigma_{X_{1}}=0.1$, while expected returns of Asset 2 follow a distribution with $\mu_{X_{2}}=0.01$ and $\sigma_{X_{2}}=0.02$. We consider a portfolio with equal weights in both assets $w_{1}=w_{2}=0.5$ and uncorrelated returns. On the left-hand side, we illustrate the behavior of $D D^{*}$ for an ex ante determined skewness coefficient varying between $-0.8 \leq$ skew $\leq 0$. On the right-hand side, we illustrate the behavior of $D D^{*}$ for an ex ante determined kurtosis between $3 \leq k u r t \leq 13$. Expected returns, standard deviation, portfolio weights and the coefficient of correlation are held constant.

weights in both assets where $w_{1}=w_{2}=0.5$ and they exhibit uncorrelated returns. We relax the assumption of Gaussian returns and consider skewed and leptokurtic asset returns. In each case we simulate 1,000,000 random numbers for Asset 1 and Asset 2 for different levels of skewness and kurtosis, while expected returns, standard deviation, portfolio weights, and the coefficient of correlation are held constant. Exhibit 5 illustrates the results for $D D^{*}$ for the assets and the generated portfolio. The left panel illustrates the behavior of $D D^{*}$ for an ex ante determined coefficient of skewness varying between 0 and -0.8 . Note that a negative coefficient of skewness refers to returns that are skewed to the left, i.e., have a higher probability to provide more extreme negative outcomes in comparison to a symmetric distribution. We observe that when asset returns become increasingly left-skewed, lower values for $D D^{*}$ are observed, indicating that the diversification of the portfolio is reduced. Values of $D D^{*}$ range from $D D^{*}=0.15$ for skew $=0$ to $D D^{*}=0.10$ for skew $=-0.8$ for the 
equal-weighted portfolio. ${ }^{6}$ The right-hand panel illustrates the behavior of $D D^{*}$ for an ex ante determined kurtosis between 3 and 13. A kurtosis of 3 is equal to the kurtosis of the Gaussian distribution. We observe that when asset returns become increasingly leptokurtic, the diversification potential of the portfolio is slightly reduced and $D D^{*}$ decreases. Values of $D D^{*}$ range from $D D^{*}=0.15$ for kurt $=3$ to $D D^{*}=0.1458$ for $k u r t=13$.

Our results indicate that for the considered equal-weighted portfolio, changes in the skewness seem to affect the proposed $D D^{*}$ more than increased kurtosis does. We also notice that in comparison to the impact of changes in the asset correlation (illustrated in Exhibit 3), the effects are clearly smaller. However, the diversification potential of the portfolio as measured by $D D^{*}$ is still significantly reduced for returns that are left-skewed or leptokurtic.

In a final step we examine $D D^{*}$ and SR optimal portfolios for situations in which asset returns become increasingly volatile and leptokurtic as typically happens during a crisis or market turmoil. Given that $D D^{*}$ also takes into account higher moments of the return distribution, we would expect a different behavior for $D D^{*}$ in comparison to the SR. We are especially interested in comparing the asset allocation for portfolios that optimize the SR and $D D^{*}$.

For the analysis we consider the same two risky assets as before: expected returns of Asset 1 again are distributed with $\mu_{X_{1}}=0.05$ and $\sigma_{X_{1}}=0.1$, while expected returns of Asset 2 follow a distribution with $\mu_{X_{2}}=0.01$ and $\sigma_{X_{2}}=0.02$. The correlation between the returns initially equals $\rho=0.3$. Now assume that due to a crisis period, the volatility for both assets increases, while at the same time asset returns become more correlated. We also assume that returns of the more risky Asset 1 become increasingly skewed and leptokurtic, while the returns for Asset 2 remain symmetric with only the volatility increasing.

To implement these changes of the market environment in our simulation study, we stepwise increase the correlation between the asset returns from $\rho=0.3$ to $\rho^{c}=0.5$, while at the same time we also increase the volatility for Asset 1 from initially $\sigma_{X_{1}}=0.1$ to $\sigma_{X_{1}}^{c}=0.2$ and for Asset 2 from $\sigma_{X_{2}}=0.02$ to $\sigma_{X_{2}}^{c}=0.06$. Finally, we also stepwise

${ }^{6}$ Similar results for $D D^{*}$ could be observed for asset returns that are skewed to the right, i.e., for an ex ante determined coefficient of skewness varying between 0 and 0.8 . However, we report results for left-skewed asset returns only, since we believe investors are more worried about the risk of extremely negative returns. 
decrease the skewness of Asset 1 returns from $s k e w_{X_{1}}=0$ to $s k e w_{X_{1}}^{c}=-0.7$ and increase its kurtosis from $\operatorname{kurt}_{X_{1}}=3$ to $\operatorname{kurt}_{X_{1}}^{c}=10$, while we assume that returns from Asset 2 remain symmetric.

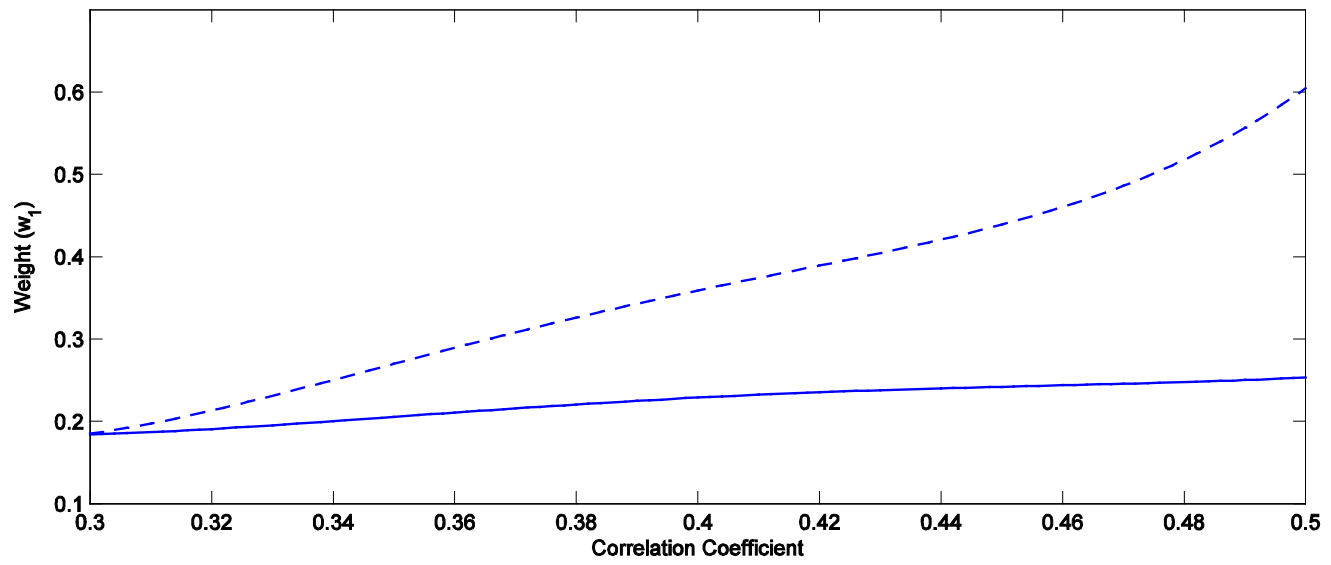

Exhibit 6: Weight $w_{1}$ given to Asset 1 for Sharpe ratio optimal (dashed) and $D D^{*}$-optimal (solid) portfolios under different market scenarios. Expected returns of Asset 1 are distributed with $\mu_{X_{1}}=0.05$, while expected returns of Asset 2 follow a distribution with $\mu_{X_{2}}=0.01$. The correlation between the asset returns is stepwise increased from $\rho=0.3$ to $\rho^{c}=0.5$, while at the same time, the volatility for Asset 1 stepwise increases from initially $\sigma_{X_{1}}=0.1$ to $\sigma_{X_{1}}^{c}=0.2$, for Asset 2 from $\sigma_{X_{2}}=0.02$ to $\sigma_{X_{2}}^{c}=0.06$, skewness and kurtosis of Asset 1 returns stepwise change from skew $_{X_{1}}=0$ to skew $_{X_{1}}^{c}=-0.7$, and from $k_{u r t}=3$ to kurt $_{X_{1}}^{c}=10$, respectively.

In each simulation step, we generate 1,000,000 correlated returns for Asset 1 and Asset 2 and then calculate the portfolio weights $w_{1}$ and $w_{2}$ that maximize the SR and $D D^{*}$, i.e., we determine the SR and $D D^{*}$ optimal portfolios. Given the worsening market conditions of increased volatility for both assets and higher correlation between the asset returns, we find that both the SR and the diversification potential decline. The SR for the optimal portfolio gradually decreases from approximately 0.62 under the original scenario to 0.26 for the crisis scenario, while $D D^{*}$ gradually decreases from 0.19 to 0.12 . So both measures are reduced, showing the worsening performance of constructed portfolios with regard to the SR and diversification under the changed market conditions.

However, examining Exhibit 6, we find that under the scenarios, SR and $D D^{*}$ optimal portfolios provide quite different results regarding asset allocation. Recall that optimal SR portfolios take into account only the first and second moment of the portfolio distribution. Thus, for $\mu_{X_{1}}=0.05$ and $\mu_{X_{2}}=0.01$ and $\sigma_{X_{1}}$ rising from its initial value of $\sigma_{X_{1}}=0.1$ to 
$\sigma_{X_{1}}^{c}=0.2$, respectively, $\sigma_{X_{2}}$ from $\sigma_{X_{2}}=0.02$ to $\sigma_{X_{2}}^{c}=0.06$, the optimal portfolio according to the SR suggests an increased investment in Asset 1. We find $w_{1}$ stepwise increases from approximately $w_{1}=0.17$ to $w_{1}=0.59$ in the crisis scenario with higher asset correlation, volatility and increased skewness and kurtosis for Asset 1. This is not surprising because the standard deviation of expected returns doubles (from $\sigma_{X_{1}}=0.1$ to $\sigma_{X_{1}}^{c}=0.2$ ) for Asset 1, while it actually triples (from $\sigma_{X_{2}}=0.02$ to $\sigma_{X_{2}}^{c}=0.06$ for Asset 2. Thus, portfolios with higher weights in Asset 1 will yield a higher SR under the changed market conditions.

On the other hand, $D D^{*}$ also considers higher moments of the return distribution of the individual assets and the portfolio. Thus, while Asset 2 provides an increasingly unfavorable SR in comparison to Asset $1, D D^{*}$ also takes into account that returns of Asset 1 become increasingly more skewed and leptokurtic. While the correlation and volatility for both assets increases, we assume that also skewness and kurtosis of Asset 1 returns stepwise increase from skew $_{X_{1}}=0$ to $s k e w_{X_{1}}^{c}=-0.7$, and from $\operatorname{kurt}_{X_{1}}=3$ to $\operatorname{kurt}_{X_{1}}^{c}=10$, respectively. Based on the fact that Asset 1 returns also exhibit increased negative skewness and kurtosis, we find only a relatively minor increase in allocations to Asset 1 for $D D^{*}$-optimal portfolios from approximately $w_{1}=0.17$ under the original scenario to $w_{1}=0.25$ under the changed market conditions.

Overall, our results illustrate how changed market conditions for correlation, volatility, and higher moments of the return distribution impact the proposed $D D^{*}$ measure. Furthermore, we show that portfolios that are constructed to maximize the SR may suggest quite different asset allocations in comparison to $D D^{*}$-optimal portfolios. As demonstrated, this difference in asset allocation can be attributed to the fact that $D D^{*}$ also considers higher moments of the return distribution of the individual assets and the portfolio, while the SR takes into account only the first and second moments of the return distribution. Therefore, in future work we recommend further analysis of the empirical performance of the $D D^{*}$ regarding portfolio optimization. 


\section{Conclusion}

Vermorken et al. (2012) introduce a new measure of diversification, the DD, based on Shannon entropy of financial returns for individual assets or a portfolio. Entropy as a measure of uncertainty has been used successfully in several frameworks and takes into account the uncertainty related to the entire statistical distribution and not just the first two moments of a distribution. We illustrate that the originally proposed DD measure has some drawbacks, in particular when risky assets such as equities are combined with asset classes with a lower risk profile.

We propose a revised measure $D D^{*}$ that is based on exponential entropy which overcomes some of the identified shortcomings of the original DD metric. This study demonstrates the properties of this new entropy-based statistic and illustrates the usefulness of the revised $D D^{*}$ measure. The results show that the revised $D D^{*}$ measure can be applied as an alternative criterion to construct optimally diversified portfolios. Portfolios that are optimal with respect to maximizing $D D^{*}$ can yield very different asset allocations compared to portfolios that are constructed by optimizing the SR. This is true in particular when empirical asset returns are asymmetric and exhibit excess kurtosis. Based on our findings, we recommend the entropy based $D D^{*}$ as an alternative measure for portfolio optimization in institutional asset management.

\section{Endnotes}

We would like to thank the Centre for International Finance and Regulation (CIFR) for providing funding support to this project under grant number SUP-005. 


\section{References}

Artzner, P., F. Delbaen, J.M. Eber, and D. Heath. "Coherent Measures of Risk." Mathematical Finance, Vol. 9 (1999) ,pp. 203-228.

Bera, A.K., and S.Y. Park. "Optimal Portfolio Diversification Using the Maximum Entropy Principle." Econometric Reviews, Vol. 26, No. 4-6 (2008), pp. 484-512.

Brown, S., and W. Goetzmann. "Hedge Funds with Style." The Journal of Portfolio Management, Vol. 29, No. 2 (2003), pp. 101-112.

Campbell, L. "Exponential Entropy as a Measure of Extent of a Distribution." Z. Wahrscheinlichkeitstheorie verw., Vol. 5 (1966), pp. 217-225.

Cover, T.M., and J.A. Thomas. Elements of Information Theory. New York, NY: John Wiley and Sons, 1991.

Dey, S., and S. Juneja. "Incorporating Fat Tails in Financial Models Using Entropic Divergence Measures." Working Paper, Tata Institute of Fundamental Research, 2012.

Dopfel, F. "Asset Allocation in Lower Stock-Bond Correlation Environment." The Journal of Portfolio Management, Vol. 30, No. 1 (2003), pp. 25-38.

Föllmer, H., and T. Knispel. "Entropic Risk Measures: Coherence vs. Convexity, Model Ambiguity, and Robust Large Deviations." Stochastics and Dynamics, Vol. 11, No. 3 (2011), pp. 1-19.

Hua, L., and L. Xingsi. "A New Portfolio Model and Application." Operations Research and Management Science, Vol. 6 (2003), pp. 83-86.

Kitamura, Y., and M. Stutzer. "An Information-Theoretic Alternative to Generalized Method of Moments Estimation." Econometrica, Vol. 65, No. 4 (1997), pp. 861-874.

Laeven, R.J.A., and M. Stadje. "Entropy Coherent and Entropy Convex Measures of Risk." Mathematics of Operations Research, Vol. 38, No. 12, (2012).

Markowitz, H.M. "Portfolio Selection." The Journal of Finance, Vol. 7, No. 1 (1952), pp. 77-91.

Maasoumi, E. "A Compendium to Information Theory in Economics and Econometrics." Econometric Reviews, Vol. 12, No. 2 (1993), pp. 137-181.

McNeil, .A, R. Frey and P. Embrechts. Quantitative Risk Management: Concepts, Techniques and Tools. New Jersey, NJ: Princeton Series in Finance, 2005.

Meucci, A. "Managing Diversification." Risk, Vol. 22, No. 5 (2009), pp. 74-79. 
Philippatos, G.C., and C. Wilson. "Entropy, Market Risk and the Selection of Efficient Portfolios." Applied Economics, Vol. 4 (1972), pp. 209-220.

Rachev, S., C. Menn, and F. Fabozzi. “Risk Measures and Portfolio Selection.” In F. Fabozzi, ed., Encyclopedia of Financial Models, Volume III, New York, NY: John Wiley and Sons, 2012, pp.349-359.

Robertson, J., E. Tallman and C. Whiteman. "Forecasting Using Relative Entropy." Journal of Money, Credit and Banking, Vol. 37, No. 3 (2005), pp. 383-401.

Rockafellar, R.T., S. Uryasev, and M. Zabarankin. "Generalized Deviations in Risk Analysis." Finance and Stochastics , Vol. 10 (2006), pp. 51-74.

Rudin, M., and J. Morgan. "A Portfolio Diversification Index." The Journal of Portfolio Management, Vol. 32, No. 2 (2006), pp. 81-89.

Shannon, C. "A Mathematical Theory of Communication." Bell System Technical Journal, Vol. 27. (1948), pp. 379-423.

Sharpe, W.F. "Mutual Fund Performance." Journal of Business, Vol. 39, No. 1 (1966), pp. 119-138.

Statman, M., and J. Scheid. "Correlation, Return Gaps, and the Benefits of Diversification." The Journal of Portfolio Management, Vol. 34, No. 3 (2008), pp. 132-139.

Stowell, D., and M. Plumbley. "Fast Multidimensional Entropy Estimation by k-d Partitioning." IEEE Signal Processing Letters, Vol. 16, No. 6 (2009), pp. 537-540.

Tasche, D. "Measuring Sectoral Diversification in an Asymptotic Multifactor Framework." Journal of Credit Risk, Vol. 2, No. 3 (2006), pp. 33-55.

Urbanowicz, K., P. Richmond, and J.A. Holyst. "Entropy and Optimization of Portfolios." Working Paper, Warsaw University of Technology, 2014.

Ullah, A. "Entropy, Divergence and Distance Measures with Econometric Applications." Journal of Statistical Planning and Inference, Vol. 49 (1996), pp. 137-162.

Vermorken, M., F. Medda, and F. Schröder. "The Diversification Delta: A Higher-Moment Measure for Portfolio Diversification." The Journal of Portfolio Management, Vol. 39, No. 1 (2012), pp. 67-74.

Wrighton, J. "Practical Applications of the Diversification Delta: A Higher-Moment Measure for Portfolio Diversification." Practical Applications , Vol. 1, No. 2 (2013), pp. 1-3. 


\section{Online Supplementary Materials}

\section{Appendices}

\section{A Diversification Delta (DD) and revised Diversification Delta $\left(D D^{*}\right)$ for a bivariate} portfolio normally distributed assets

Let $P=\left(w_{1} X_{1}+w_{2} X_{2}\right)$ be a portfolio where the two assets are normal, $X_{1} \sim N\left(0, \sigma_{X_{1}}^{2}\right)$ and $X_{2} \sim N\left(0, \sigma_{X_{2}}^{2}\right)$ and the weights are positive and satisfy $w_{1}+w_{2}=1$. In this case $P \sim N\left(0, \sigma_{P}^{2}\right)$, with $\sigma_{P}^{2}=w_{1}^{2} \sigma_{X_{1}}^{2}+2 \rho w_{1} w_{2} \sigma_{X_{1}} \sigma_{X_{2}}+w_{2}^{2} \sigma_{X_{2}}^{2}$, where $\rho$ is the correlation between the assets.

Note that the entropy of a normally distributed variable $X$, with variance $\sigma_{X}^{2}$, is $\log \left(\sqrt{2 \pi e \sigma_{X}^{2}}\right.$ ) (see Cover and Thomas (1991)). Therefore, considering equations (1) and (3), the Diversification Delta $(D D)$ and the revised Diversification Delta $\left(D D^{*}\right)$ in this bivariate normal case are

$$
\begin{aligned}
D D(P) & =\frac{\exp \left(w_{1} \log \left(\sqrt{2 \pi e \sigma_{X_{1}}^{2}}\right)+w_{2} \log \left(\sqrt{2 \pi e \sigma_{X_{2}}^{2}}\right)\right)-\exp \left(\log \left(\sqrt{2 \pi e \sigma_{P}^{2}}\right)\right)}{\exp \left(w_{1} \log \left(\sqrt{2 \pi e \sigma_{X_{1}}^{2}}\right)+w_{2} \log \left(\sqrt{2 \pi e \sigma_{X_{2}}^{2}}\right)\right)} \\
& =\frac{\left[\exp \left(\log \left(\sqrt{2 \pi e} \sigma_{X_{1}}\right)\right]^{w_{1}} \cdot\left[\exp \left(\log \left(\sqrt{2 \pi e} \sigma_{X_{2}}\right)\right)\right]^{w_{2}}-\sqrt{2 \pi e} \sigma_{P}\right.}{\left[\exp \left(\log \left(\sqrt{2 \pi e} \sigma_{X_{1}}\right)\right)\right]^{w_{1}} \cdot\left[\exp \left(\log \left(\sqrt{2 \pi e} \sigma_{X_{2}}\right)\right)\right]^{w_{2}}} \\
& =\frac{\left(\sqrt{2 \pi e} \sigma_{X_{1}}\right)^{w_{1}} \cdot\left(\sqrt{2 \pi e} \sigma_{X_{2}}\right)^{w_{2}}-\sqrt{2 \pi e} \sigma_{P}}{\left(\sqrt{2 \pi e} \sigma_{X_{1}}\right)^{w_{1}} \cdot\left(\sqrt{2 \pi e} \sigma_{X_{2}}\right)^{w_{2}}} \\
& =\frac{(\sqrt{2 \pi e})^{w_{1}+w_{2}} \cdot \sigma_{X_{1}}^{w_{1}} \sigma_{X_{2}}^{w_{2}}-\sqrt{2 \pi e} \sigma_{P}}{(\sqrt{2 \pi e})^{w_{1}+w_{2}} \cdot \sigma_{X_{1}}^{w_{1}} \sigma_{X_{2}}^{w_{2}}} \\
& =\frac{\sigma_{X_{1}}^{w_{1}} \sigma_{X_{2}}^{w_{2}}-\sigma_{P}}{\sigma_{X_{1}}^{w_{1}} \sigma_{X_{2}}^{w_{2}}} \\
& =1-\frac{\sigma_{P}}{\sigma_{X_{1}}^{w_{1}} \sigma_{X_{2}}^{w_{2}}}
\end{aligned}
$$




$$
\begin{aligned}
D D^{*}(P) & =\frac{w_{1} \exp \left(\log \left(\sqrt{2 \pi e \sigma_{X_{1}}^{2}}\right)\right)+w_{2} \exp \left(\log \left(\sqrt{2 \pi e \sigma_{X_{2}}^{2}}\right)\right)-\exp \left(\log \left(\sqrt{2 \pi e \sigma_{P}^{2}}\right)\right)}{w_{1} \exp \left(\log \left(\sqrt{2 \pi e \sigma_{X_{1}}^{2}}\right)\right)+w_{2} \exp \left(\log \left(\sqrt{2 \pi e \sigma_{X_{2}}^{2}}\right)\right)} \\
& =\frac{w_{1} \sqrt{2 \pi} e \sigma_{X_{1}}+w_{2} \sqrt{2 \pi} e \sigma_{X_{2}}-\sqrt{2 \pi} e \sigma_{P}}{w_{1} \sqrt{2 \pi} e \sigma_{X_{1}}+w_{2} \sqrt{2 \pi} e \sigma_{X_{2}}} \\
& =\frac{\left(w_{1} \sigma_{X_{1}}+w_{2} \sigma_{X_{2}}\right)-\sigma_{P}}{\left(w_{1} \sigma_{X_{1}}+w_{2} \sigma_{X_{2}}\right)} \\
& =1-\frac{\sigma_{P}}{\left(w_{1} \sigma_{X_{1}}+w_{2} \sigma_{X_{2}}\right)}
\end{aligned}
$$

\section{B Original Diversification Delta for Example 3}

Investor 1 constructs a portfolio from assets $X_{1}$ and $X_{2}$. This investor determines that a portfolio with equal weights is optimal, i.e. $P_{1}=\frac{1}{2} X_{1}+\frac{1}{2} X_{2}$. In a different market, investor 2 constructs a portfolio from assets $Y_{1}=\frac{3}{2} X_{1}$ and $Y_{2}=\frac{3}{4} X_{2} \cdot{ }^{7}$ This investor determines optimal weights of $w_{1}=\frac{1}{3}$ and $w_{2}=\frac{2}{3}$, yielding the same portfolio $P_{2}=\frac{1}{2} X_{1}+\frac{1}{2} X_{2}$.

Let $c_{1}=\exp \left(H\left(X_{1}\right)\right), c_{2}=\exp \left(H\left(X_{2}\right)\right)$ and $c_{3}=\exp \left(H\left(\frac{1}{2} X_{1}+\frac{1}{2} X_{2}\right)\right)$. Given that the portfolios are the same, from equation (1) in both cases we have

$$
\begin{aligned}
D D(P) & =\frac{\exp \left(w_{1} H\left(Y_{1}\right)+w_{2} H\left(Y_{2}\right)\right)-\exp (H(P))}{\exp \left(w_{1} H\left(Y_{1}\right)+w_{2} H\left(Y_{2}\right)\right)} \\
& =\frac{\exp \left(w_{1} H\left(Y_{1}\right)+w_{2} H\left(Y_{2}\right)\right)-c_{3}}{\exp \left(w_{1} H\left(Y_{1}\right)+w_{2} H\left(Y_{2}\right)\right)} .
\end{aligned}
$$

Error! Bookmark not defined.In the case of Portfolio 1,

$$
\begin{aligned}
\exp \left(w_{1} H\left(Y_{1}\right)+w_{2} H\left(Y_{2}\right)\right) & =\exp \left(\frac{1}{2}\left(H\left(X_{1}\right)+H\left(X_{2}\right)\right)\right) \\
& =\left[\exp \left(H\left(X_{1}\right)+H\left(X_{2}\right)\right)\right]^{\frac{1}{2}} \\
& =\left(c_{1} C_{2}\right)^{\frac{1}{2}},
\end{aligned}
$$

and for Portfolio 2

\footnotetext{
${ }^{7}$ Note that the coefficients in these equations represent volume not weights.
} 


$$
\begin{aligned}
\exp \left(w_{1} H\left(Y_{1}\right)+w_{2} H\left(Y_{2}\right)\right) & =\exp \left(\frac{1}{3} H\left(\frac{2}{3} X_{1}\right)+\frac{2}{3} H\left(\frac{3}{4} X_{2}\right)\right) \\
& =\left[\exp \left(H\left(\frac{3}{2} X_{1}\right)\right)^{\frac{1}{3}} \cdot\left[\exp \left(H\left(\frac{3}{4} X_{2}\right)\right)\right]^{\frac{2}{3}}\right. \\
& =\left[\frac{3}{2} \exp \left(H\left(X_{1}\right)\right)\right]^{\frac{1}{3}} \cdot\left[\frac{3}{2} \frac{\exp \left(H\left(X_{2}\right)\right)}{2}\right]^{\frac{2}{3}} \\
& =\left(\frac{3}{2}\right)^{\frac{1}{3}} c_{1}^{\frac{1}{3}} \cdot\left(\frac{3}{2}\right)^{\frac{2}{3}}\left(\frac{c_{2}}{2}\right)^{\frac{2}{3}} \\
& =\left(\frac{3}{2}\right) \cdot\left(\frac{c_{1} c_{2}^{2}}{4}\right)^{\frac{1}{3}} \cdot
\end{aligned}
$$

Therefore, we get

$$
D D\left(P_{1}\right)=\frac{\left(c_{1} c_{2}\right)^{\frac{1}{2}}-c_{3}}{\left(c_{1} c_{2}\right)^{\frac{1}{2}}} \neq D D\left(P_{2}\right)=\frac{\frac{2}{3}\left(\frac{c_{1} c_{2}^{2}}{4}\right)^{\frac{1}{3}}-c_{3}}{\frac{3}{2}\left(\frac{c_{1} c_{2}^{2}}{4}\right)^{\frac{1}{3}}} .
$$

\section{Revised Diversification Delta for combination of identical assets}

Let $P$ be a portfolio consisting of assets $\left(X_{1}, \ldots, X_{N}\right)$ which are all positive linear combinations of an asset $X$. Hence, $X_{i}=a_{i} X+b_{i}$, for constants $a_{i}>0$ and $b_{i}$ with $i \in\{1, \ldots, N\}$.

$$
\begin{gathered}
\sum_{i=1}^{N} w_{i} \exp \left(H\left(X_{i}\right)\right)=\sum_{i=1}^{n} w_{i} a_{i} \exp (H(X))=\exp (H(X)) \sum_{i=1}^{n} w_{i} a_{i}=\exp \left(H\left(\sum_{i=1}^{n} w_{i} a_{i} X\right)\right) \\
=\exp \left(H\left(\sum_{i=1}^{n} w_{i} a_{i} X+\sum_{i=1}^{n} w_{i} b_{i}\right)\right)=\exp \left(H\left(\sum_{i=1}^{n} w_{i} X_{i}\right)\right),
\end{gathered}
$$

Therefore,

$$
D D^{*}(P)=\frac{\sum_{i=1}^{N} w_{i} \exp \left(H\left(X_{i}\right)\right)-\exp \left(H\left(\sum_{i=1}^{N} w_{i} X_{i}\right)\right)}{\sum_{i=1}^{N} w_{i} \exp \left(H\left(X_{i}\right)\right)}=0
$$




\section{Revised Diversification Delta for Example 3}

Again, let $c_{1}=\exp \left(H\left(X_{1}\right)\right), c_{2}=\exp \left(H\left(X_{2}\right)\right)$ and $c_{3}=\exp \left(H\left(\frac{1}{2} X_{1}+\frac{1}{2} X_{2}\right)\right)$. Using (3), in both cases we have

$$
\begin{aligned}
D D^{*}(P) & =\frac{w_{1} \exp \left(H\left(Y_{1}\right)\right)+w_{2} \exp \left(H\left(Y_{2}\right)\right)-\exp (H(P))}{w_{1} \exp \left(H\left(Y_{1}\right)\right)+w_{2} \exp \left(H\left(Y_{2}\right)\right)} \\
& =\frac{w_{1} \exp \left(H\left(Y_{1}\right)\right)+w_{2} \exp \left(H\left(Y_{2}\right)\right)-c_{3}}{w_{1} \exp \left(H\left(Y_{1}\right)\right)+w_{2} \exp \left(H\left(Y_{2}\right)\right)}
\end{aligned}
$$

For Portfolio 1,

$$
w_{1} \exp \left(H\left(Y_{1}\right)\right)+w_{2} \exp \left(H\left(Y_{2}\right)\right)=\frac{1}{2}\left(\exp \left(H\left(X_{1}\right)\right)+\exp \left(H\left(X_{2}\right)\right)\right)=\frac{1}{2}\left(c_{1}+c_{2}\right),
$$

and for Portfolio 2

$$
\begin{aligned}
w_{1} \exp \left(H\left(Y_{1}\right)\right)+w_{2} \exp \left(H\left(Y_{2}\right)\right) & =\frac{1}{3} \exp \left(H\left(\frac{3}{2} X_{1}\right)+\frac{2}{3} H\left(\frac{3}{4} X_{2}\right)\right) \\
& =\frac{1}{3} \times \frac{3}{2} \exp \left(H\left(X_{1}\right)\right)+\frac{2}{3} \times \frac{3}{4} \exp \left(H\left(X_{2}\right)\right) \\
& =\frac{1}{2}\left(c_{1}+c_{2}\right) .
\end{aligned}
$$

\section{Error! Bookmark not defined.}

Therefore, we obtain

$$
D D^{*}\left(P_{1}\right)=D D^{*}\left(P_{2}\right)=\frac{c_{1}+c_{2}-2 c_{3}}{c_{1}+c_{2}}
$$

